\title{
LA CIUDAD COMO SUJETO Y OBJETO DE APRENDIZAJE SOCIAL, ESTÉTICO Y EMOCIONAL \\ Christof Göbel*
}

RESUMEN: Las ciudades son centros de comunicación y de aprendizaje en las cuales se concentra la energía física, intelectual y creativa. Mediante instalaciones e intervenciones urbanas en el espacio público de ciudades en México y Alemania, se experimentó con el concepto de aprobación, entendido según la teoría de apropiación de Alexej N. Leontiev como sinónimo de un proceso específico de aprendizaje. Aprender del otro y con el otro, así como aprender del lugar, convierten el espacio público de la ciudad en un lugar de aprendizaje y, con ello, de identidad que deja huellas en la memoria de las personas.

yose

The City as SubJect And OBJect of Social, Aesthetic And EMotional LeARning

ABSTRACT: Cities are centers of communication and learning, where physical, intellectual and creative energy is concentrated. Through urban installations and interventions in the public space of cities in Mexico and Germany, the task of approval was approached in an experimental manner, understood according to Alexej N. Leontiev's appropriation theory as a synonym of a specific learning process. Learning from and with the other, as well as learning from the place, turns the public space of the city into a place of learning and identity, leaving traces in the memory of the people.

PALABRAS CLAVE: experimentación, apropiación, espacio público.

KEY WORDS: experimentation, appropriation, public space.

RECEPCIÓN: 6 de noviembre de 2016.

APROBACIÓN: 14 de agosto de 2018.

* Universidad Autónoma Metropolitana, Azcapotzalco. 
Se prohíbe su reproducción total o parcial por cualquier medio, incluido electrónico, sin permiso previo y por escrito de los editores. 


\section{LA CIUDAD COMO SUJETO Y OBJETO \\ DE APRENDIZAJE SOCIAL, ESTÉTICO Y EMOCIONAL}

\section{Introducción}

La ciudad, como ente de máximo valor cultural y de diseño, representa un lugar de aprendizaje de competencias sociales, estéticas y emocionales, lo cual implica una ampliación del sentido de formación en general, así como se expone en diversos contextos pedagógicos, pensando en un aprendizaje constante. De este modo, no solo se aprende en los institutos tradicionales, como la escuela o la universidad; se aprende en la ciudad, según el lema universal de la "ciudad como salón de clases", tomando en cuenta la existencia de lugares de aprendizajes múltiples y el espacio público en particular.

En el marco de la teoría de apropiación de Alexei N. Leontiev, se pretende mostrar en qué manera el espacio público como elemento importante de la ciudad surte un efecto de aprendizaje de competencias diversas en la gente, utilizando el método de instalaciones e intervenciones urbanas experimentales. 


\section{La teoría de apropiación de Alexei N. Leontiev}

"Apropiación" es un término multifacético y emblemático que alude a las ideas de ocupación, adquisición, expropiación, adueñamiento, privación, usurpación o conquista. Igualmente, se utiliza para denotar un proceso específico socioemocional de aprendizaje o como equivalente de un aprendizaje subjetivo por identificación. El aprendizaje es una construcción propia del conocimiento y del sentido para la vida, y contribuye a la formación de identidad como una apropiación emocional. Por último, la apropiación también se refiere a la adquisición de formas humanas de comportamiento.

Michel de Certeau escribió: "Los lugares se convierten en espacios a través de la apropiación y su uso concreto", ${ }^{1}$ refiriéndose a una interpretación de espacio orientada a la acción. La teoría de apropiación planteada por el psicólogo ruso Leontiev señala que "el mundo real que más determina la vida humana es un mundo que ha sido transformado por la actividad humana. Como un mundo de objetos sociales que simbolizan las habilidades humanas formadas durante el transcurso de la práctica histórica social, no son dadas al individuo en forma inmediata, ya que en estas propiedades se presentan como una tarea para cada ser humano", ${ }^{2}$ lo que significa que, al contrario de los animales que aprenden primeramente por herencia o adaptación, los seres humanos observan y aprenden por imitación; por ejemplo, si un niño ve que su papá come la sopa con la cuchara en posición horizontal para que la sopa no se derrame, está observando, codificando, decodificando y aprendiendo a través de un proceso de adaptación. Así, el ser humano aprende, mientras que la apropiación se entiende como una confrontación activa con el medio ambiente.

Las condiciones de las vidas externas son productos de trabajo humano. En ello están acumulados los logros (experiencias, significados) del trabajo histórico social de manera figurativa. Esta cosificación de facultades humanas y de trabajo humano en sus productos se enfrenta ahora a la apropiación como parte individual del proceso de producción

${ }^{1}$ Michel de Certeau, Kunst des Handelns, 1988, Berlín, Suhrkamp.

${ }^{2}$ Alexei Nikolajewitsch Leontiev, Probleme der Entwicklung des Psychischen, 1980, Königstein, Athenäum, p. 281. 
figurativo. Por lo tanto, cosificación y apropiación aparecen como dos caras de un mismo proceso sociohistórico.

El ser humano aprende por apropiación de manera procesual, lo que lleva a una extensión del ámbito de la acción. La apropiación es un proceso en el que se reproducen los rasgos históricamente formados, las aptitudes y los comportamientos; no ocurre en el vacío, sino en el medio ambiente. La apropiación se lleva a cabo en el espacio (Figura 1). "Porque los espacios — como escribe Ulrich Deinet—, especialmente los espacios urbanos, no han sido inalterados naturalmente, sino elaborados por los seres humanos, diseñados, modificados y construidos, hay que apropiárselos, lo mismo que los significados que contienen, tal como se hace con los objetos y con las herramientas del entorno inmediato."3 Por ello, en el sentido objetivo y personal, la apropiación es en el espacio urbano también una apropiación espacial figurativa.

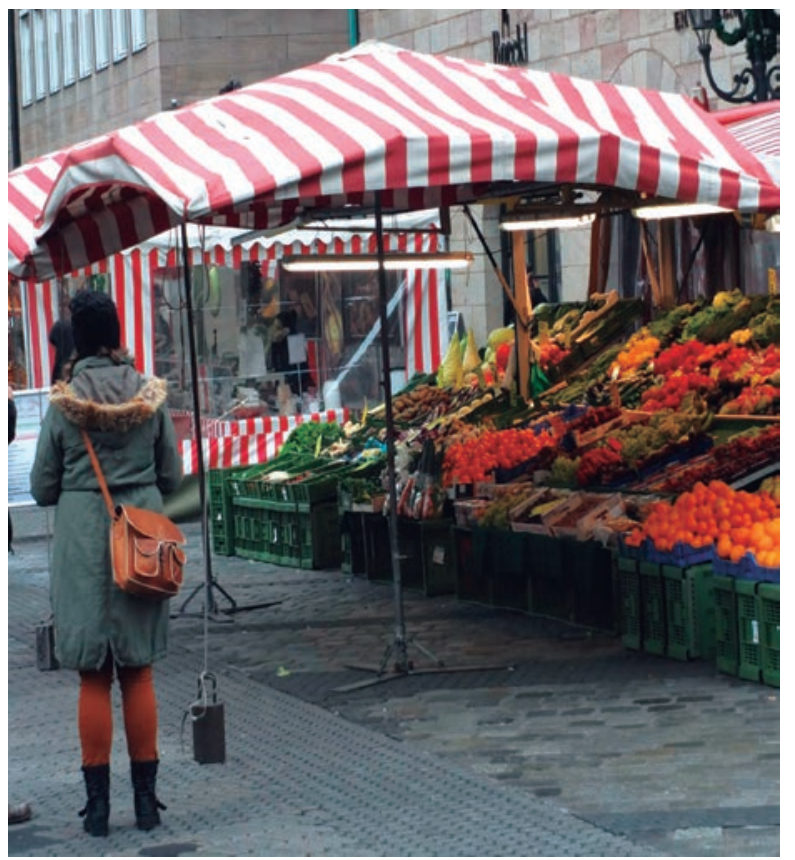

Figura 1. Apropiación espacial en el mercado central en Núremberg, Alemania. Fotografía: Christof Göbel.

${ }^{3}$ Ulrich Deinet (comp.), Sozialräumliche Jugendarbeit. Grundlagen, Methoden und Praxiskonzepte, 2009, Wiesbaden, Verlag für Sozialwissenschaften, pp. 27-58. 


\section{El espacio público y la ciudad}

Desde siempre han existido en las ciudades lugares centrales, en los cuales la gente se reúne y comercia, es decir, donde se "hace ciudad", socializando e intercambiando económica, cultural y afectivamente. Los espacios públicos son puntos focales de la vida pública, y por lo tanto, representan un tema central, un importante elemento espacial de la ciudad. Jordi Borja explica que "el espacio público es el símbolo de la ciudad en sí misma", por lo cual, de alguna manera se establece un equilibrio entre la ciudad y el espacio público (Figura 2).

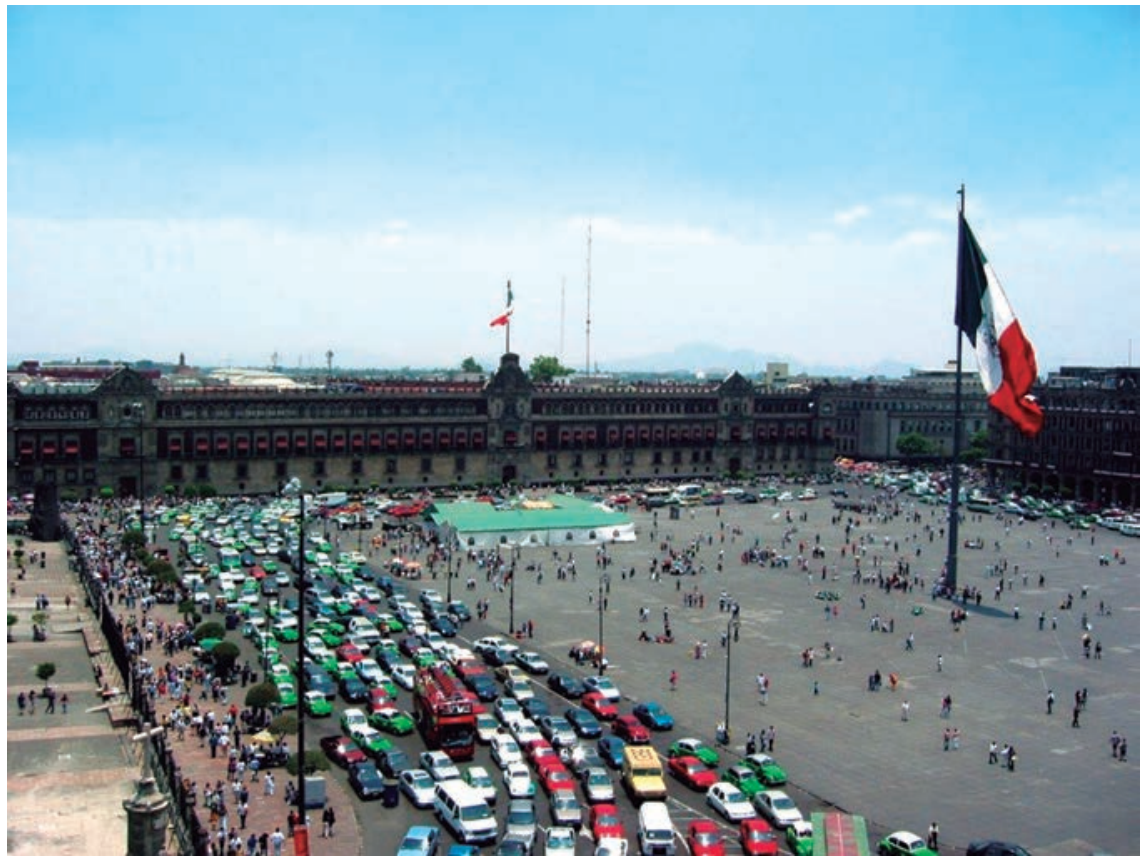

Figura 2. El Zócalo o Plaza de la Constitución como representación de la Ciudad de México.

Fotografía: Cortesía de Daniel Escotto Sánchez, coordinador general de la Autoridad del Espacio Público en el Gobierno de Marcelo Ebrard.

La ciudad no es solo un fenómeno del diseño, sino más bien una expresión física de la estructuración de espacio y tiempo, es decir: es la estructuración de la vida comunitaria, llevando inmediatamente a la noción 
de lugares de la acción comunitaria; de tal manera, el espacio público es un producto de la sociedad y le sirve como escenario público. Por lo tanto, el espacio público no es meramente un "contenedor" físicamente comprensible, tridimensional, sino un "entorno" para la acción social, es decir, el espacio siempre es el resultado de acciones específicas. Los espacios de acción existen solo como unidad inseparable con las personas que operan en ellos. Así, el espacio público es un sitio donde cada uno encuentra un lugar y está caracterizado por una sociedad diversa, basada en las diferencias. Ya que también la ciudadanía se constituye primeramente a través de las prácticas urbanas, como manifiesta Sergio Tamayo ${ }^{4}$ en su concepto de los "espacios para la ciudadanía", podría decirse que en estas formas de socialización urbana, la función de la sociedad civil se (re) territorializa.

El espacio público supone un dominio público, un uso social colectivo y una multifuncionalidad, y se caracteriza físicamente por su accesibilidad y la calidad de las relaciones sociales. La importancia del espacio público para la ciudad es relevante, pues en él se pueden observar aspectos económicos, sociales y culturales que dan sentido a las prácticas de una sociedad y de una ciudad encarnadas en el desarrollo de la identidad urbana. Parafraseando a Jordi Borja, "la mejor manera de medir la calidad de una ciudad es por su espacio público o por sus implicaciones políticas". 5

De tal manera, el espacio público de muchas ciudades como la Ciudad de México se ve reducido, restringido. Hoy se habla a menudo de una "crisis de ciudad y de urbanidad" (Figura 3), ya que con las transformaciones de la forma y la estructura urbana, también han cambiado el carácter, uso y apropiación del espacio público. Nuevos espacios de socialización emergen especialmente en la periferia, los cuales refuerzan los procesos de segregación urbana y legitiman la represión de la construcción y el mantenimiento de estos modelos de socialización.

${ }^{4}$ Sergio Tamayo, "Die Stadt und die Produktion des Ciudadanía-Raums. Eine stadtsoziologische Perspektive", en Huffschmid, A. y Wildner, K. (comps.), Stadtforschung in Lateinamerika. Neue urbane Szenarien: Öffentlichkeit-Territorialität-Imaginarios, 2013, Bielefeld, Transcript Verlag, pp. 203-223.

5ordi Borja, "Ciudadanía y espacio público", Ambiente y Desarrollo, XIV/3 (1998), pp.13-22. 
La dimensión pública está cambiando por un sistema de espacios colectivos (Figura 4) en los que las nuevas tipologías se basan en el comportamiento del consumidor y son determinadas por el deseo de seguridad pública. Los espacios se utilizan como espacios públicos que, en sentido estricto, no son públicos, más bien seudopúblicos, aunque la mayoría de la gente los percibe como tales.

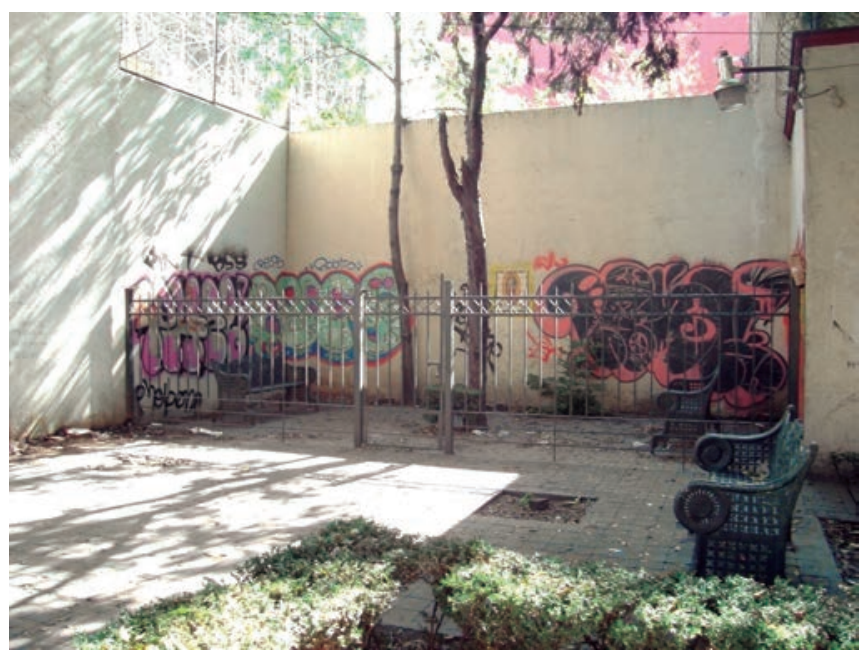

Figura 3. Crisis del espacio público. Fotografía: Cortesía de Daniel Escotto Sánchez.

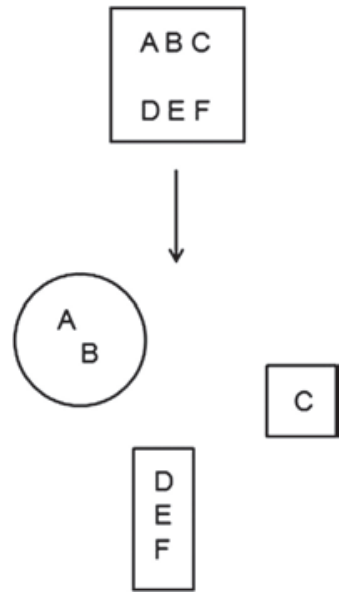

Figura 4. Representación de la transformación del espacio público en la Ciudad de México. 
Teóricamente, el espacio se refiere a una estructura flexible y negociable definida por la organización espacial. ${ }^{6}$ Por ello, el espacio ha dejado de definirse como una unidad pasiva, como un "buque" tridimensional euclídico, y debe entenderse como un proceso entre el entorno físico y la práctica social discursiva. Es decir, la idea del espacio público como resultado de procesos sociales de producción cambia constantemente. De acuerdo con Lefebvre, ${ }^{7}$ el espacio está pensado, construido y usado entablando una relación entre lo físico y lo social, mientras que se reconoce el cambio de perspectiva o de paradigma por un espacio generado social y culturalmente en las ciencias culturales como "cambio topológico" o spatial turn, refiriéndose a la relación del tiempo y del espacio que lleva a un modelo no absoluto, sino espacial relacional. ${ }^{8}$

De la misma manera se puede interpretar a la arquitectura como dinámica, más allá de un marco material, físico, a la cual se le exige, además, que proporcione situaciones espaciales apropiadas para el movimiento y la acción. Por ejemplo, una ventana ofrece la oportunidad de abrirla, de mirar afuera, así como de ventilar el espacio, por lo cual la arquitectura tiene la capacidad de crear situaciones. Asimismo, la radio bocina montada en la Plaza Aguilita en el barrio de la Merced en el centro histórico de la Ciudad de México utiliza ciertos elementos arquitectónicos, como una mesa, sillas o los mismos altavoces, de modo que representa ese arreglo arquitectónico en el sentido amplio (Figura 5). "El papel crucial de la arquitectura significa en este contexto la interacción entre las características espaciales de los elementos estructurales y de las condiciones en las cuales puedan percibirse, utilizarse y experimentarse." 9 El espacio se produce cuando la arquitectura está en uso, y no por la forma arquitectónica en sí. Por otra parte, se describe a la arquitectura como un espacio abierto a la intervención, en el que la colectividad o

${ }^{6}$ Kathrin Wildner, Zócalo-Die Mitte der Stadt Mexiko. Ethnographie eines Platzes, 2013, Berlín, D. Reimer Verlag, p. 58.

${ }^{7}$ Cfr. Henri Lefebvre, La production de l'espace, 1974, París, Anthropos.

${ }^{8}$ Martina Löw, Raumsoziologie, 2001, Fráncfort del Meno, Suhrkamp.

${ }^{9}$ Alban Janson y Florian Tigges, Grundbegriffe der Architektur. Das Vokabular räumlicher Situationen, 2013, Basilea, Birkhäuser Verlag. 
la compartición de la ciudad sirven como base para repensar temas sociales en términos urbanísticos. "La ciudad es nuestro potencial y somos sus creadores." 10

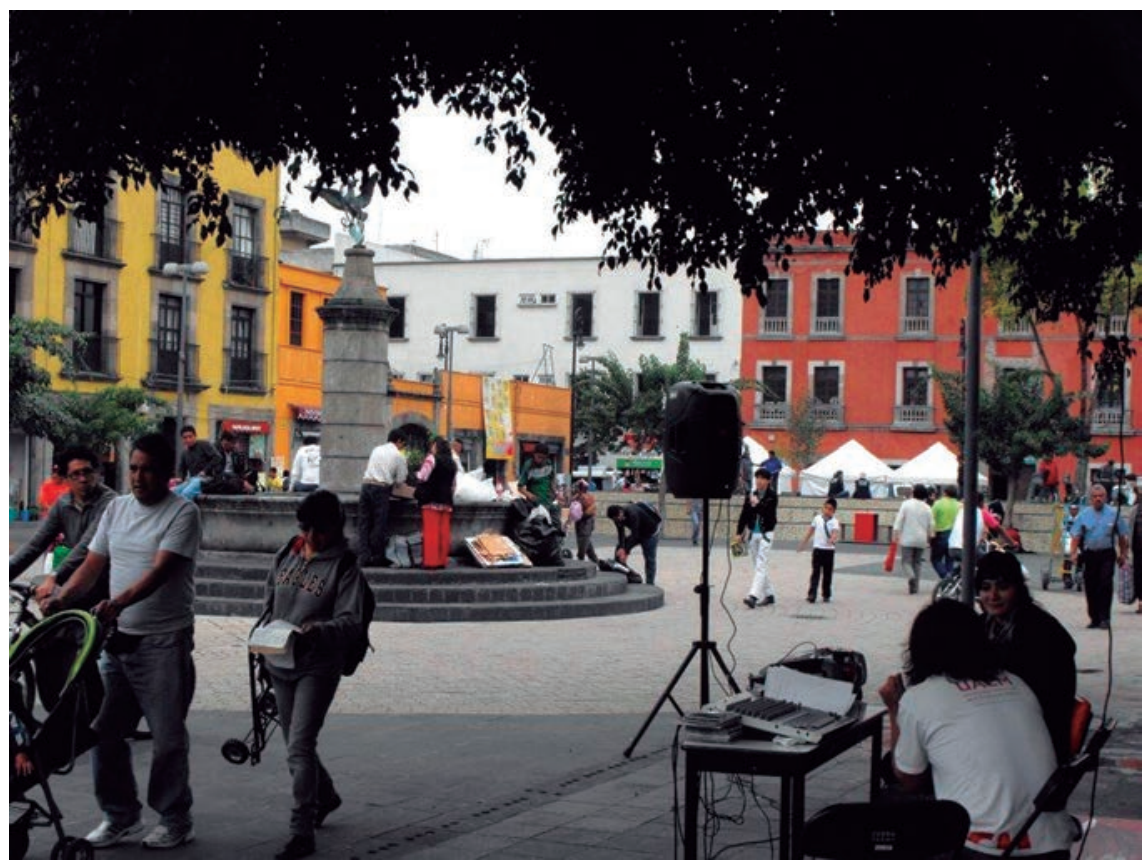

Figura 5. Situación arquitectónica producida por la Radio Aguilita.

Fotografía: Cortesía de Joaquín Aguilar Camacho, promotor cultual de la Casa Talavera, Universidad Autónoma de la Ciudad de México.

En este sentido, se muestran a continuación algunos proyectos de intervenciones o instalaciones urbanas en el espacio público en la Ciudad de México y en Alemania. Estos proyectos se acercan a la tarea de apropiación de forma experimental y promueven la apropiación como sinónimo de aprendizaje social, estético y emocional. Partiendo de la tesis de que las características de una sociedad como componentes de la identidad se proyectan en el espacio, se entienden las características de un espacio público como una representación de la ciudad misma. Así, los experimentos pretendieron crear percepciones y servir

\footnotetext{
${ }^{10}$ Raumlabor Berlin, Acting in public, 2008, Berlín, Jovis Verlag.
} 
de reflexión, interpretando el espacio público como un medio a través del cual pueden formularse ideas sobre la ciudadanía y la pertenencia a una ciudad.

\section{Experimentación en el espacio público de la ciudad}

Fair Trade, Fototeca, Ciudad de México, 2008

En el marco de SITIOS.08, el primer taller internacional de SITIOS, un laboratorio del espacio público, se proyectó, entre otras, la intervención urbana Fair Trade (Figura 6), que se llevó a cabo junto con estudiantes de la Universidad Técnica de Darmstadt, Alemania, la Universidad Técnica de Graz, Austria, y de la Universidad Autónoma Metropolitana, Azcapotzalco, en la Ciudad de México. SiTıOs es una red internacional e interdisciplinaria en la interfaz entre arte, arquitectura y estudios urbanos que busca soluciones urbanas con respecto al espacio público.

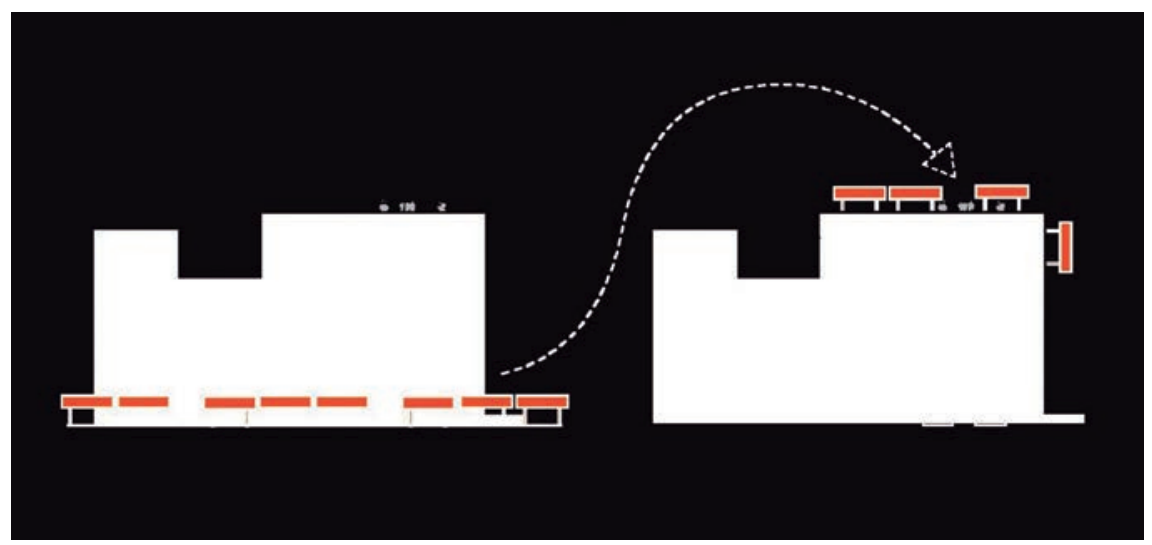

Figura 6. Concepto de la instalación o intervención urbana propuesta. Fotografía: Cortesía de SITIOS.

En el techo de la Fototeca, un archivo fotográfico en el centro histórico de la Ciudad de México, se propuso mediante una escultura social un mercado temporal de ambulantes (Figura 7), cuidando también el efecto estético, espacial y social, con la intención de ofrecer un foro 
de discusión sobre el problema de la expulsión de los vendedores ambulantes antes de las elecciones políticas. Aunque al final el proyecto no se llevó a cabo, la acción sirvió para crear conciencia sobre la virulenta apropiación del espacio público urbano por parte de sistemas comerciales, que representa una realidad recurrente en la megalópolis del país.

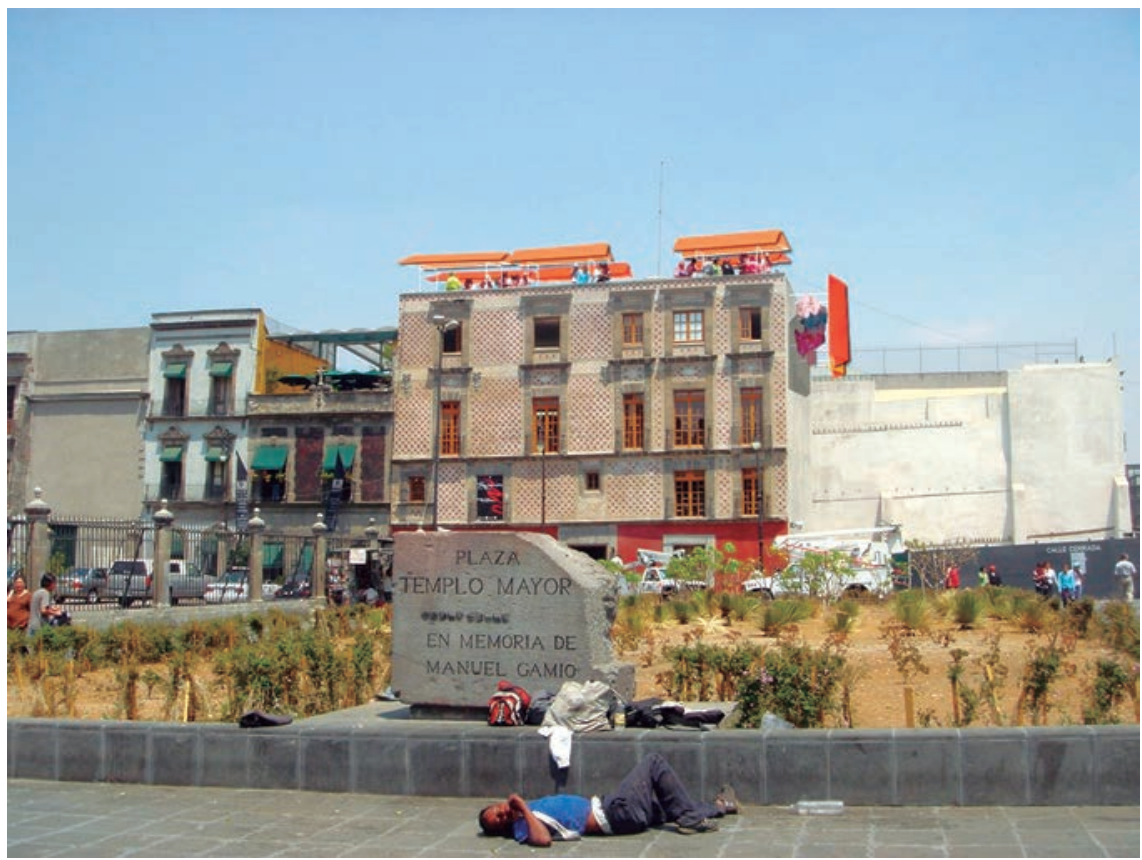

Figura 7. Fotomontaje de la escultura social propuesta por SITIOS. Fotografía: Cortesía de sitios.

Tapar para Ver, Plaza San Luca, Ciudad de México, 2012

Otros alumnos de la carrera de arquitectura de la UAM, Azcapotzalco investigaron, en el marco del "Taller de experimentación en el espacio público", la Plaza San Luca en la Colonia Centro, a un costado del Metro Pino Suárez, y encontraron un espacio público sectorizado, sucio y en desorden. Entonces, dividieron esta plaza de manera figurativa en 
tres zonas: una de comercio, la plaza y un espacio levemente hundido que llamaron "Plaza de los Pobres", tomando en cuenta que la fragmentación y segregación es un reflejo cada vez más marcado en la Ciudad de México. Mientras que las otras dos zonas bullían de gente como comerciantes y transeúntes, nadie se atrevía a pisar la Plaza de los Pobres, aparte de algunos indigentes aislados.

Este sector está mal iluminado, y además, al estar encajonado por mucha vegetación, no se ventila naturalmente y transmite una sensación de inseguridad. Con el fin de crear conciencia y estimular la transformación de este espacio residual, el grupo elaboró a mano una cortina de papel reciclado, para tapar esta zona y verla con ojos distintos después de su redescubrimiento (Figura 8). Esa acción in situ del grupo resultó bastante atractiva y fue interpretada por la gente como un "encanto para los ojos", en referencia a la parte estética del lugar. Es decir, la reacción de quienes se acercaron a este lugar mostró que la intervención urbana dejó una huella en la percepción de la gente.

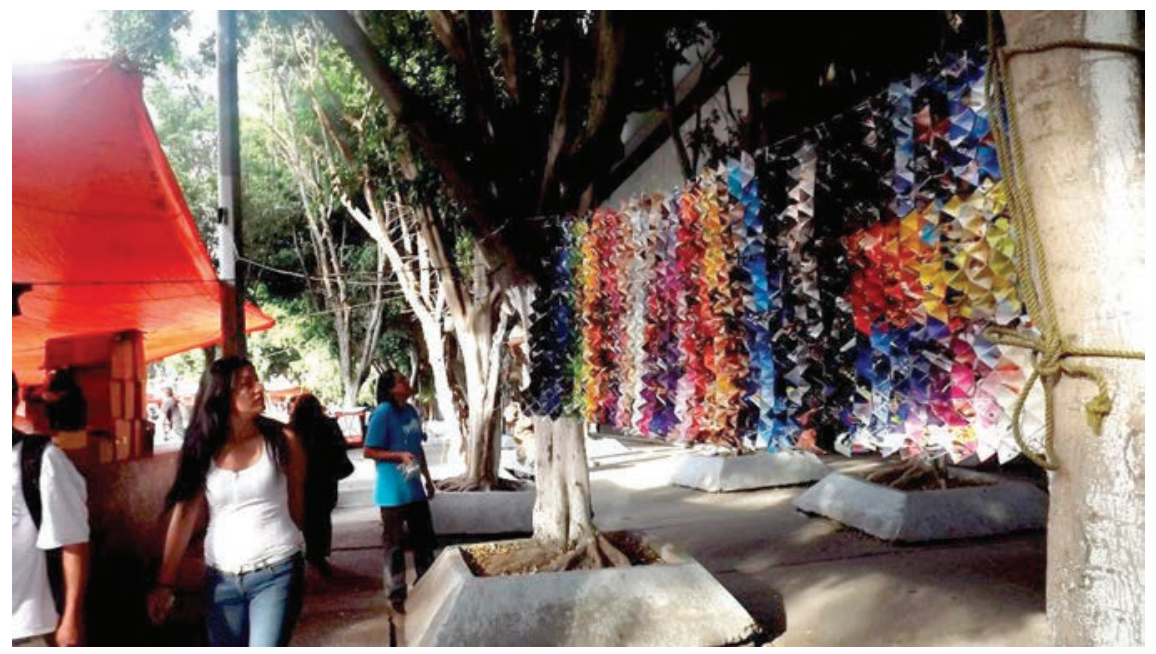

Figura 8. Pasantes admirando la cortina de papel reciclado elaborado por alumnos de la UAM. Fotografía: Cortesía de Silvana G. Cid Prado. 
La Gran Reinauguración del Tláloc de Tlatelolco, Ciudad de México, 2013

Otro experimento se realizó en Tlatelolco, a partir del descubrimiento de un monumento a Tláloc, que había estado olvidado y deteriorado (Figura 9). La zona donde se encuentra no es visitada. Ahora hay iniciativas para reubicar la escultura en otro espacio. Antes de que avanzara el deterioro, se organizó con alumnos de la especialidad y maestría en Estudios Urbanos del Posgrado en Diseño/CyAD, llamado Grupo de Estudios Urbanos Alternativos, como parte del Taller de Diseño III, una reinauguración de este monolito con el fin de "revivirlo", reactivar la zona donde se encuentra y nutrir de valor simbólico a la Unidad Nonoalco-Tlatelolco.

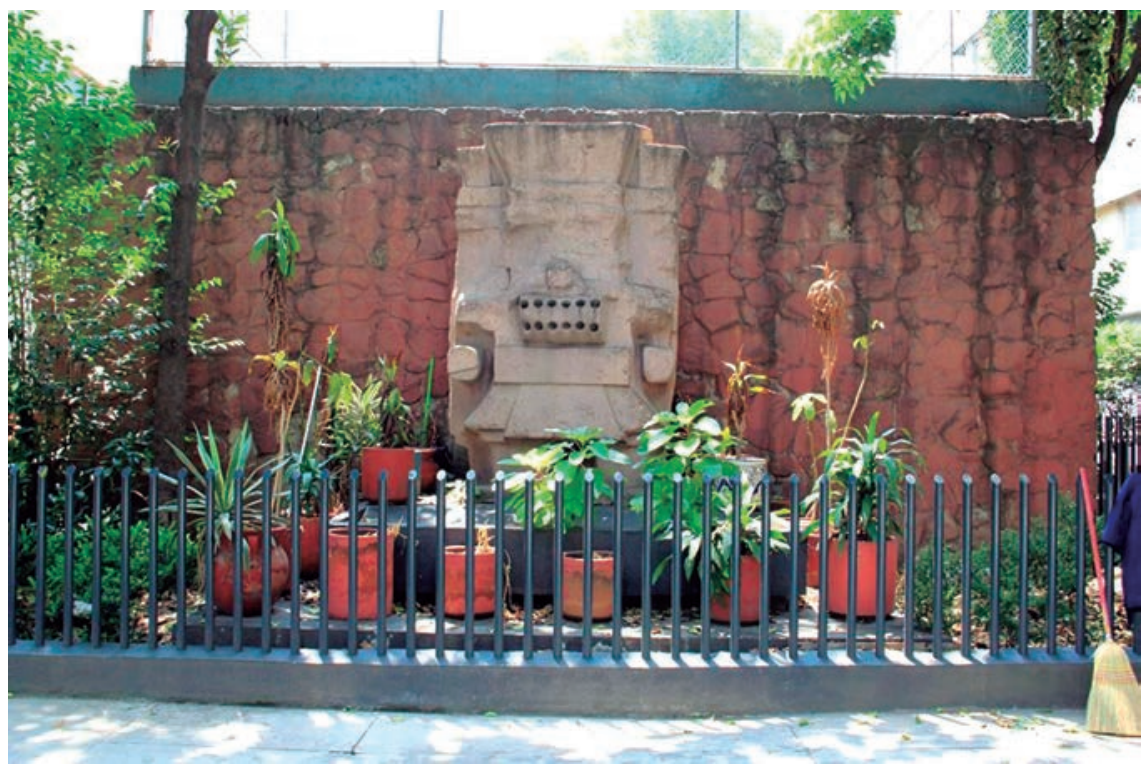

Figura 9. La estatua de Tláloc antes de la intervención.

Fotografía: Cortesía del Grupo de Estudios Urbanos Alternativos.

La reinauguración se llevó a cabo el 13 de julio de 2013 a las 11:30 horas (Figura 10). Participaron representantes de Coatlinchán, pueblo del Estado de México, en cuyas cercanías se encontraba el monolito ori- 
ginal de Tláloc que fue trasladado en abril de 1964 a la Ciudad de México y que hoy está en la entrada del Museo Nacional de Antropología. Los habitantes de Coatlinchán compartieron su sabiduría y experiencia, y efectuaron un ritual en honor a este monumento, del que revelaron que en realidad se trataba de Chalchiuhtlicue, la diosa de los lagos y corrientes de agua, también conocida por sus pobladores como la "Piedra de los Tecomates". Así, se hizo un recuento de la historia de la escultura. Fueron invitados representantes de diversos países, se develó una placa conmemorativa y al final se ofreció una degustación de antojitos mexicanos.

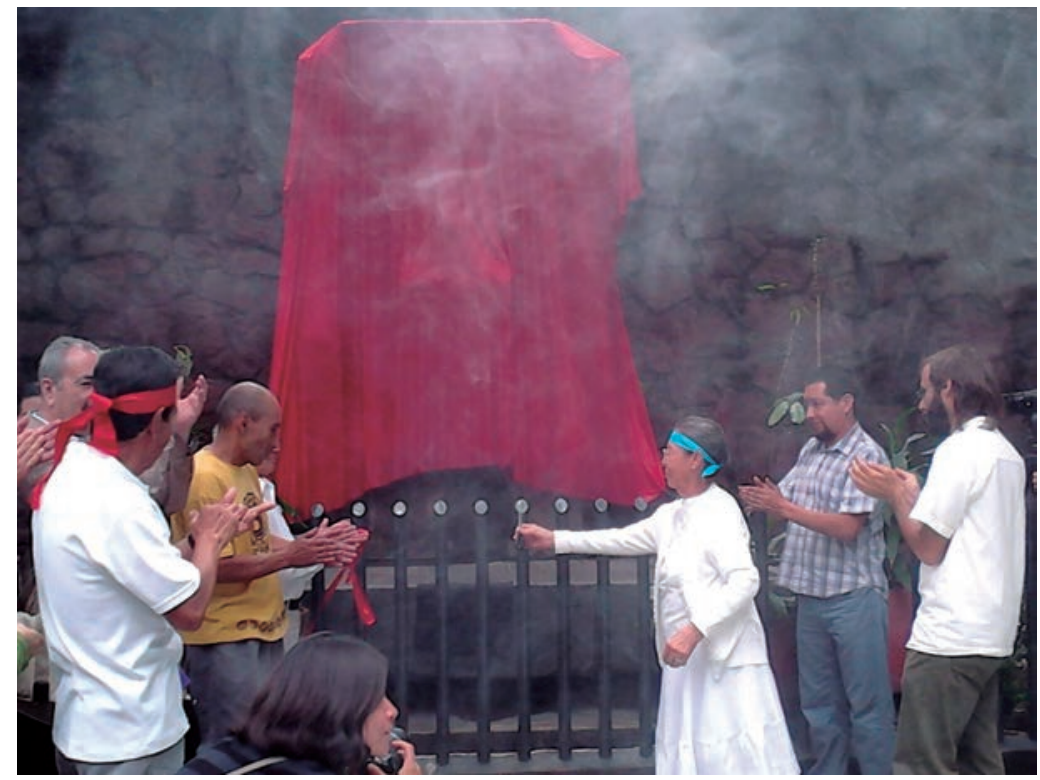

Figura 10. Reinauguración del Tláloc de Tlatelolco con habitantes de Coatlinchán. Fotografía: Cortesía del Grupo de Estudios Urbanos Alternativos.

Los estudiantes esperaron que esta reinauguración se convirtiera en un punto de partida para que los vecinos de Tlatelolco visibilizaran y cuidaran ellos mismos un espacio que casi nadie conocía. Este tipo de proyectos tienen que ver con la visualización, con la manera de vivir un lugar, con la estética y con la práctica social, y esto le da cierto sentido a la vida, a la pertinencia del lugar. 
Waldplastik ("Bosque sintético”), plaza del mercado central en Núremberg, Alemania, 2014

Cada año desde 2000, en el mes de mayo se lleva a cabo en el casco antiguo de Núremberg, Alemania, un evento denominado la "Noche Azul" (Blaue Nacht), en el que el centro histórico se convierte en un mar de luces, sonidos y baile, con artistas y alumnos seleccionados en el campo del arte y diseño. Edificios, casas, plazas y patios están adornados por proyecciones e instalaciones. La prominente plaza del mercado central ("Hauptmarkt") de Núremberg — uno de los escenarios - fue iluminado este año por la instalación estudiantil Waldplastik. La instalación artística fue desarrollada por el Instituto de Arquitectura del Paisaje y Espacio Público (dirigido por la profesora Regine Keller) de la Universidad Técnica de Múnich, y fue el proyecto más estético de los presentados.

El centro de la intervención es el mercado. La plaza del mercado central de Núremberg acoge de lunes a sábado un mercado de frutas y verduras. Ese mercado se caracteriza por ser plano y sin árboles. Se distingue por los puestos y las sombrillas del mercado que determinan su identidad. En los días del mercado, se reparten sobre la plaza hasta 80 puestos (Figura 11).

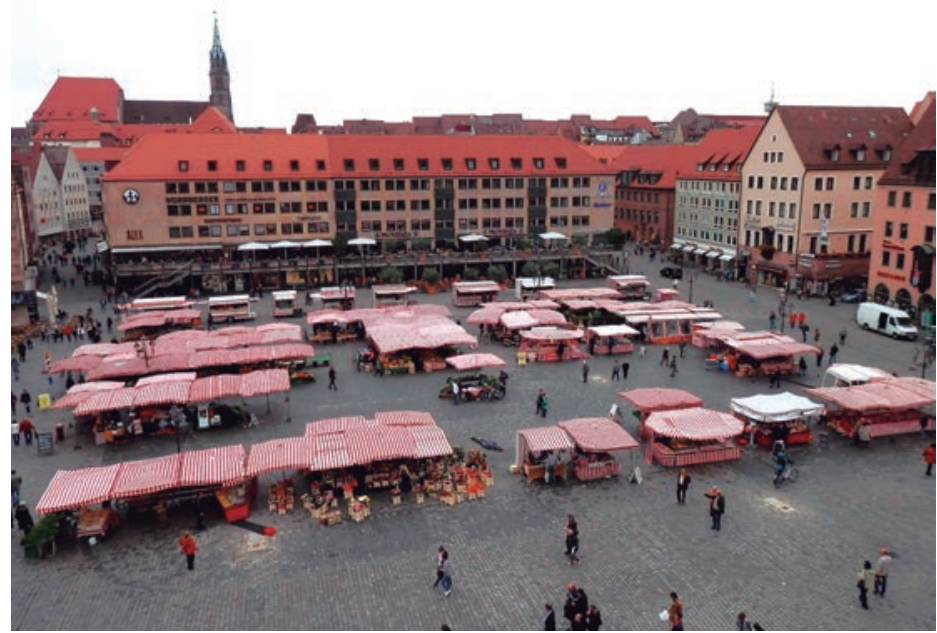

Figura 11. Plaza del mercado central, Núremberg. Fotografía: Christof Göbel. 
Con el lema "cuando el mercado hizo una caminata por el bosque", los estudiantes de Arquitectura del Paisaje lograron que se "trajera el bosque a la plaza" reinterpretando el mercado de frutas y verduras en un bosque mediante una instalación espacial de iluminación sobre los materiales del mercado cotidiano y árboles artificiales. Se transformaron los paraguas del mercado en troncos, las bolsas del mercado en follaje y las cajas de frutas en asientos, de modo que el bosque de plástico se convirtió en una escultura del bosque. Los estudiantes interpretaron críticamente el lema de la Noche Azul 2014, "anhelo”, basando el concepto de su propuesta en el ideal del bosque como un lugar nostálgico que incita sensaciones místicas, románticas, aventureras, melancólicas o felices y transformaron el mercado de un espacio de rutina en un lugar de deseo. Así lograron una atmósfera fantástica, con una estructura forestal entre los temas característicos del mercado.

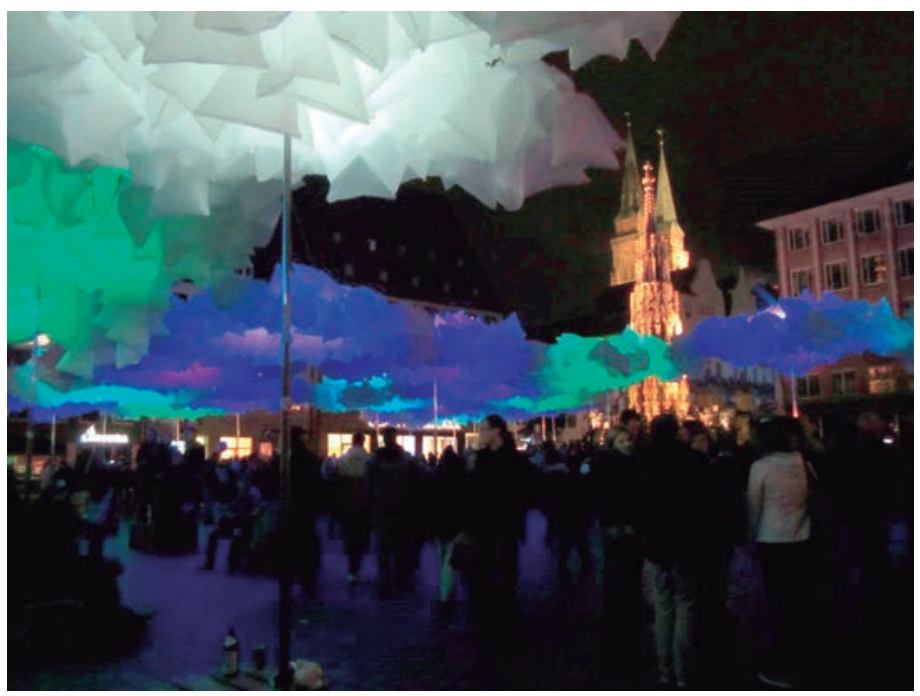

Figura 12. Intervención artística realizada por estudiantes de la Universidad Técnica de Múnich, 3 de mayo de 2014. Fotografía: Cortesía del Instituto de Arquitectura del Paisaje y Espacio Público, Múnich, Alemania.

El uso del contexto aparentemente trivial de los acontecimientos del mercado convierte este lugar de la cotidianidad en un escenario de 
lo especial. El bosque se experimenta por sus elementos separados, los cuales forman una unidad, y el mercado se vuelve tangible en su totalidad para los visitantes. Además de la estética artística inmediata (Figura 12), la instalación ofreció la oportunidad de discutir ciertos temas actuales de sentido urbanístico y medio ambiente de Núremberg, por ejemplo, el problema ecológico de las bolsas de plástico o la idea de plantar árboles en la plaza del mercado central.

\section{El espacio público como lugar de aprendizaje de la ciudad}

En conclusión, se propone el espacio público de la ciudad como un lugar de aprendizaje de competencias sociales, estéticas y emocionales. En este lugar de encuentro y de la ciudadanía se promueve la apropiación como un proceso especifico de aprendizaje subjetivo por identificación, desarrollando formas humanas de comportamiento (Figura 13).
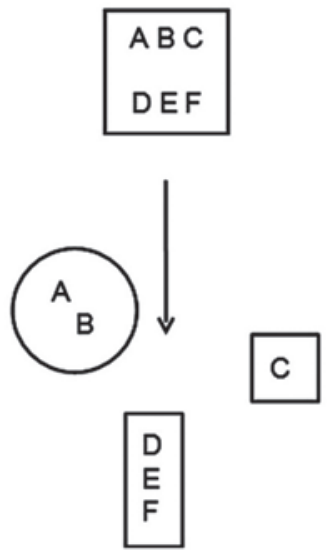

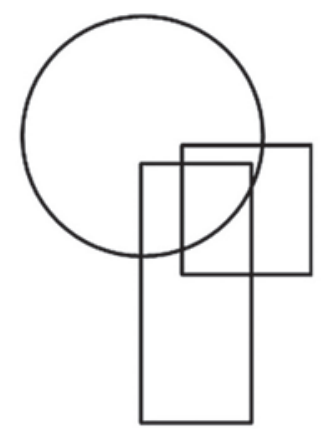

Figura 13. Idea del espacio público urbano de hoy y su función como un lugar de aprendizaje múltiple.

En el encuentro entre los artistas y los estudiantes, así como con los curiosos y transeúntes que presenciaron los proyectos, por ejemplo, se aprende del otro a tratar lo diferente y, con ello, se aprende a tolerar. Aparte de este aprendizaje y comprensión intercultural, mediante la 
interacción social se adquieren competencias como las facultades de comunicación y para crear contactos, empatía y discreción, competencias de cooperación y resolución de conflictos, valor cívico y la habilidad de la anticipación social.

De acuerdo con la idea del aprendizaje cooperativo participativo en la teoría social cognitiva de Albert Bandura ${ }^{11}$ y su concepto de "interaccionismo", el comportamiento humano no solo está determinado por los estímulos externos ni únicamente por predisposiciones cognitivas, sino que se construye a través de la interacción de situaciones en las que intervienen tanto estímulos externos como personas. El espacio público interpretado como lugar de encuentro y de la ciudadanía representa también un escenario para los conflictos entre los actores de la sociedad y, finalmente, un lugar de aprendizaje de condiciones y relaciones sociales, que promueve una actitud de comprensión cultural que abre también una oportunidad para la democracia.

Además, se acerca al genius loci al confrontarse con el lugar y sus particularidades, al relacionarlo con significados y manifestaciones emocionales y al proporcionarle simbolismos para elaborar protocolos cotidianos que dan sentido a la vida. Se aprende a apropiarse y poblar el espacio. Asimismo, la apropiación significa una extensión del espacio de acción, al remitirse a los nuevos paradigmas espaciales de la actualidad y al crear por espacio la actividad propia. Así, se considera el espacio público como temporal, performativo y participativo, en el cual las acciones los transforman de manera continua. La apropiación espacial y de la vida significa crear espacios y no solamente apropiarse figurativamente de los existentes. Es decir, por medio de la apropiación espacial se modifican y rediseñan situaciones preexistentes y arreglos, y al comprobar el repertorio extendido de comportamientos y de nuevas habilidades se desarrollan competencias prácticas que producen social, estética y emocionalmente los espacios. Al mismo tiempo, la apropiación significa movimiento o cambio, vinculación de las “islas" o entornos específicos en los que todos vivimos para ayudar a relacionarnos. Por ello, se describe el movimiento espacial también como una oscilación entre el mundo interior y exterior.

\footnotetext{
${ }^{11}$ Albert Bandura, Sozial-kognitive Lerntheorie, 1979, Stuttgart, Klett-Cotta.
} 
Aprender del lugar, aprender del otro y aprender a través de la acción común convierten el espacio público en un lugar de aprendizaje de competencias sociales, estéticas y emocionales (Figura 14), y con ello, de identidad, sentido de vida y permanencia en las ciudades. La identificación y la identidad del lugar refuerzan la idea de una memoria colectiva con el sitio al cual las intervenciones urbanas presentadas contribuyeron vinculando emocionalmente el espacio y el lugar. Este vínculo emocional, interpretado como un primer acercamiento a la apropiación del espacio, lleva en consecuencia a que la gente se sienta responsable de los espacios de la ciudad y cuide los lugares, para preservar el patrimonio material e inmaterial y reconocer el espíritu del lugar.

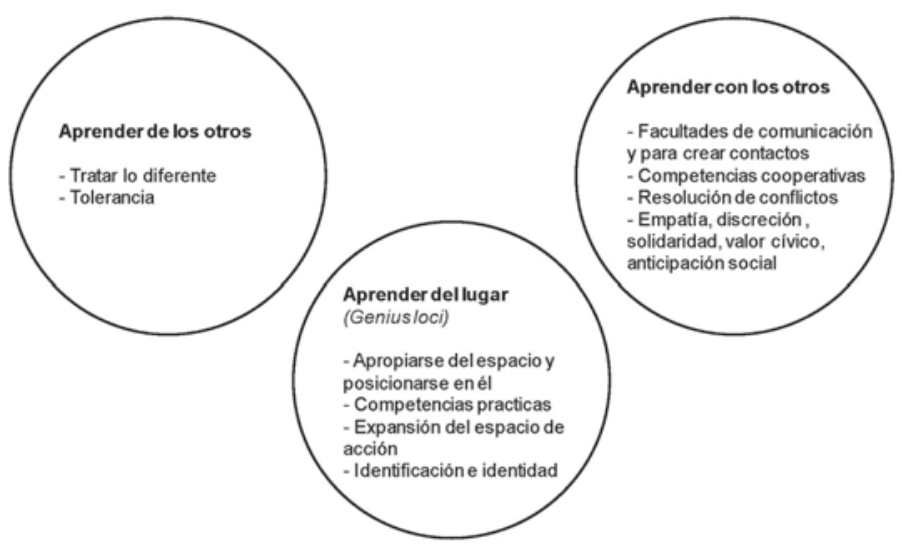

Figura 14. Esquema de aprendizaje en el espacio público de la ciudad.

Más allá de los conceptos teóricos, estos proyectos posibilitan una experiencia práctica y real de aproximación a la ciudad. Las instalaciones e intervenciones urbanas experimentales fomentan la reflexión sobre un lugar y estimulan las reacciones al espacio público de la ciudad. Se pudo observar una motivación notable para entender las características particulares del espacio tendiendo experiencias in situ a través de las cuales se podrían poner a prueba los conocimientos, ideas y la creatividad de los participantes, lo mismo que sus habilidades sociales y culturales al confrontarse con sus propias idiosincrasias y apropiarse de manera variada de los proyectos. 
Al mismo tiempo, el acercamiento emocional al lugar da valor al espacio público, aunque una megalópolis tan grande como la Ciudad de México aparente un carácter predominante de frialdad, individualidad o egoísmo. Así, los proyectos transforman a la gente y las formas de percibir la ciudad. Por ello, se considera que las instalaciones e intervenciones urbanas son un medio importante de formación experimental para enseñar a la gente las realidades espaciales y sociales que podrían obstaculizar el encuentro y la formación de la ciudadanía en la Ciudad de México, donde el espacio físico y social están separados limitando así la función del espacio público como lugar de aprendizaje.

A pesar de su carácter efímero, los proyectos dejan huellas en la memoria de la gente o llevan a una redefinición del lugar, sea de manera sutil, crítica o provocadora. Así, se crea cierta sostenibilidad que surte un efecto a mediano y largo plazo en la memoria colectiva e individual. Estos destellos de proyectos disruptivos, si bien son efímeros, ayudan a conectar al espíritu de la ciudad, al aprendizaje social, estético y emocional con la ciudad misma. Las ciudades son productos de la experiencia, es decir, que son vividas, que condensan energía física, intelectual y creativa. Por lo tanto, las ciudades representan centros de comunicación y de aprendizaje múltiples, lo que contribuye a la producción de conocimiento. 
Se prohíbe su reproducción total o parcial por cualquier medio, incluido electrónico, sin permiso previo y por escrito de los editores. 